\title{
Erratum
}

\section{Entropy Changes for \\ Steady-State Fluctuations}

R. Landauer ${ }^{2}$

Received August 16, 1974

The explanation on p. 359 is structured incorrectly. The term $\delta q_{0}$ in Eq. (10) and in the paragraph at the bottom of the page should be replaced by the ensemble average, $\langle\delta q\rangle$. The term $\delta U_{F}$ must be defined as

$$
\delta U_{F}=\delta\langle U\rangle-(d U / d q)_{q_{0}}\langle\delta q\rangle
$$

and, as recognized elsewhere in the paper, is not a perfect differential. It is not simply an energy of fluctuations. These errors are local and do not affect the remainder of the discussion. As pointed out on p. 360, for the equilibrium distribution $(d U / d q)_{\alpha_{0}}$ is identical to $\langle d U / d q\rangle$.

Section 6 of the paper contains real errors and should be ignored.

${ }^{1}$ This paper appeared in J. Stat. Phys. 9(4):351 (1973).

${ }^{2}$ IBM Thomas J. Watson Research Center, Yorktown Heights, New York. 